\title{
The morphology and filtration performances of poly(ether sulfone) membrane fabricated from different polymer solution
}

\author{
Afrillia Fahrina ${ }^{1}$, Teuku Maimun ${ }^{1}$, Syarifah Humaira ${ }^{1}$, Cut Meurah Rosnelly ${ }^{1}$, Mirna Rahmah Lubis ${ }^{1}$, Intan Bahrina, \\ Rahmat Sunarya ${ }^{1}$, Ahmad Ghufran ${ }^{1}$, and Nasrul Arahman ${ }^{1, *}$
}

${ }^{1}$ Syiah Kuala University, Chemical Engineering Department, 23111 Banda Aceh, Indonesia

\begin{abstract}
The performance of membrane filtrations are mostly determined by pore structure of the fabricated membrane. Selection of polymer and solvent in membrane preparation are influence the morphology of fabricated membrane. The present work discusses the morphology of PES membrane fabricated from different polymer system with constant preparation condition. Polymer system consists of $15 \mathrm{wt} \%$ of polyethersulfone (PES) and $85 \mathrm{wt} \%$ of solvents. The homogeneous solution includes of PESdimethyl sulfoxide (DMSO), PES-dimethylformamide (DMF), and PES-N-methylpyrrolidone (NMP). The purpose of this research is to investigate the effect of the types of solvent on the formed membrane morphology. Phase inversion technique is used to solidify the flat sheet membrane in deionized water as a coagulation bath. The scanning electron microscopy (SEM) was used to observe the morphology of membrane. A dead-end ultrafiltration module was carried out to observe the filtration performance of the fabricated membrane. It is proved that the different membrane solution affect the membrane structure in term of skin layer, macrovoid, and support layer. Furthermore, the changing of the membrane structure affected the pure water permeability (PWP). It is found that the highest PWP was reached up to 4.52 $\mathrm{L} / \mathrm{m}^{2}$.hr.atm using PES-DMSO system.
\end{abstract}

\section{Introduction}

Polyethersulfone (PES) is one of the polymer membranes often used in water purification process. The advantages of PES membrane are having good mechanical property, able to conduct in high temperature (up to $200^{\circ} \mathrm{C}$ ), and outstanding resistance with chlorine and risk chemicals, as well as its malleability into various module configurations [1-3]. The harder benzene ring and softer ether bond in the structure lead PES with crystalline structure. In membrane fabrication, an asymmetric structure plays very important role in membrane filtration performance. Several factors affecting the result of fabricated membrane are polymer composition, solvent concentration, and gelation nature [4].

This article discusses the morphology of PES membrane prepared using three different solvents, those are N-methylpyrrolidone (NMP), dimethyl sulfoxide (DMSO), and dimethylformamide (DMF). The exchange of solvent from membrane solution to nonsolvent in coagulation bath lead a formation of polymer rich phases resulted in a porous membrane [5]. Therefore, the exchange rate and interaction between solvents and nonsolvent result different structure of PES membrane. Arthanareeswaran and Starov [5] investigate the impact of different solvents on PES membrane fabrication. The result showed the PES-DMSO system has thinner top layer and big macrovoid structures. Hasbullah et al. [6] prepared PES membrane using three different solvents, NMP, DMAc, and DMF. The fabricated membrane showed PES-NMP system has the smallest pore size and highest rejection. In this study, the types of solvent investigated the result of the membrane are NMP, DMSO, and DMF.

\section{Materials and method}

Main materials used were Polyethersulfone membrane (PES) from Ultrason E6020P. N-methylpyrrolidone (NMP), dimethyl sulfoxide (DMSO), and dimethylformamide (DMF) as selected solvents. Vial bottles as a membrane casting solution media, applicator used to cast the solution in flat sheet, membrane ultrafiltration module for permeation test, and other laboratory instruments for membrane morphology and water permeability analysis.

\subsection{Fabrication of membrane}

The first step of membrane manufacturing is the preparation of polymer solution. Compositions of the solution are consist of $15 \mathrm{wt} \%$ of polyethersulfone (PES) and $85 \mathrm{wt} \%$ of solvent. The PES was dissolved into three types of solvents namely NMP, DMSO, DMF. The membranes were manufactured via non-solvent induced phase separation (NIPS) by immersion precipitation

\footnotetext{
"Corresponding author: nasrular@unsyiah.ac.id
} 
technique. When the homogenous solution was reached, the dope was then cast using an applicator onto the support media such as glass plate. The membrane film was dipped into a non-solvent solution bath that consists of water.

\subsection{Membrane characterization and filtration performance}

The characteristics of fabricated membranes were analyzed in term of membrane morphology and hydrophilicity properties. The filtration performance of membrane was investigated by using a dead-end filtration module with single flat sheet membrane. Scanning electron microscopy (SEM) was used to observe the structure of the membrane. Pure water permeability was measured to investigate the membrane performance. Membrane permeability was calculated using Eq. (1)

$L_{p}=\frac{V}{A x+\Delta_{s}}$

Where;

$\mathrm{Lp}=$ Permeability $\left(\mathrm{L} / \mathrm{m}^{2} \cdot\right.$ h.bar $)$

$\mathrm{V}=$ volume of permeat $(\mathrm{L})$

A $=$ surface area of membrane $\left(\mathrm{m}^{2}\right)$

$\Delta \mathrm{p}=$ pressure as driving force (bar)

$\mathrm{T}=$ time of filtration $(\mathrm{h})$

\section{Results and discussion}

\subsection{Membrane morphology}

The morphology of membranes affects the performance of membranes application especially for filtration process [7, 8]. Many researchers are more attracted to investigate the modification of membrane morphology in various techniques. The prominent purpose of the modification is to enhance the filtration performance and to keep the membrane life time by controlling the fouling problem. In this work, the pore structure of membrane was modified by changing the type of solvent. The morphology changes of the membrane with different of solvents are presented in Figure 1, Figure 2, and Figure 3 respectively.

Figure 1 indicates the SEM image on the top surface of membrane structure fabricated from different polymer composition. A dense structure found in PES-NMP and PES-DMF systems, while the surface of PES-DMSO system has more of porous. When the membrane film was immersed into the coagulation bath, the NMP and DMF solvent in the membrane has a lower affinity towards the water as a non solvent than DMSO. The low diffusion rate between solvent and nonsolvent leads the increasing of polymer concentration in the surface and caused delay demixing in coagulation bath. Therefore the surface of PES-NMP and PES-DMF membrane has a denser pore structure.

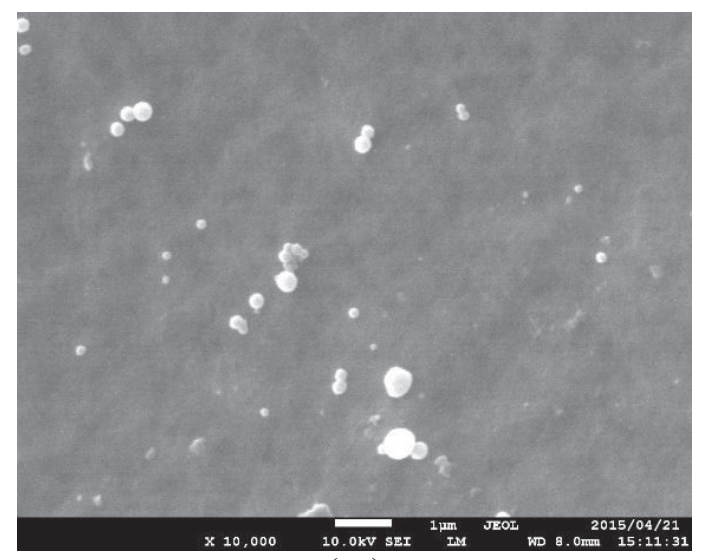

( a )

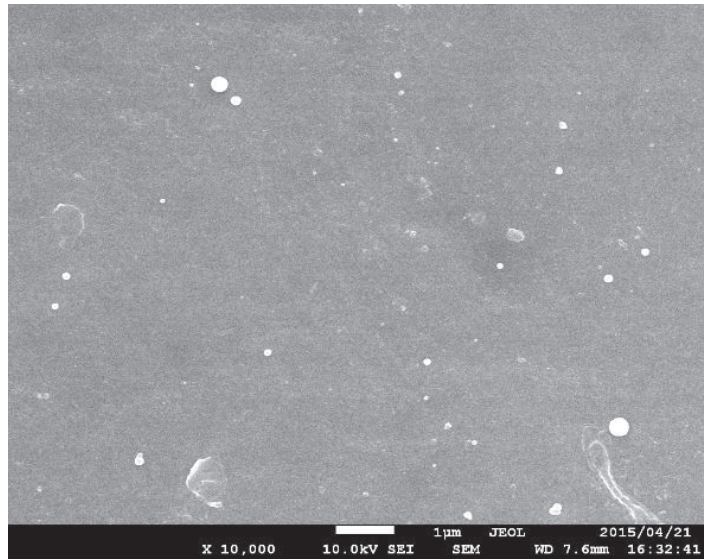

(b)

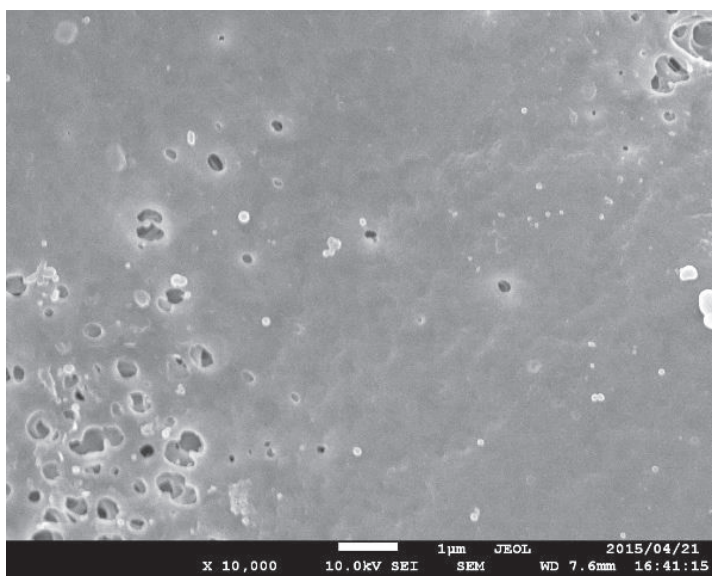

( c )

Fig. 1. SEM images of top surface membranes: PES-NMP (a); PES-DMF (b); PES-DMSO(c).

Figure 2 shows the SEM images of finger-like structure support layer taken on cross-section area of membranes prepared from PES/NMP, PES/DMF, and PES/DMSO. The all membrane modification systems produced the asymmetric structures consist of top layer and bottom as support layer. It is one of the membrane characteristics made via non-solvent induced phase separation technique. The thickest top layer found in PES/NMP system due to delay demixing when phase separation, while thin top layer produced in another system that has good affinity to nonsolvent. Based on 
morphology analysis, it can be concluded that the good interaction of solvent and nonsolvent in this study can be written as DMSO $>$ DMF $>$ NMP. Furthermore, the morphology of membranes inside macrovoid was also evaluated.

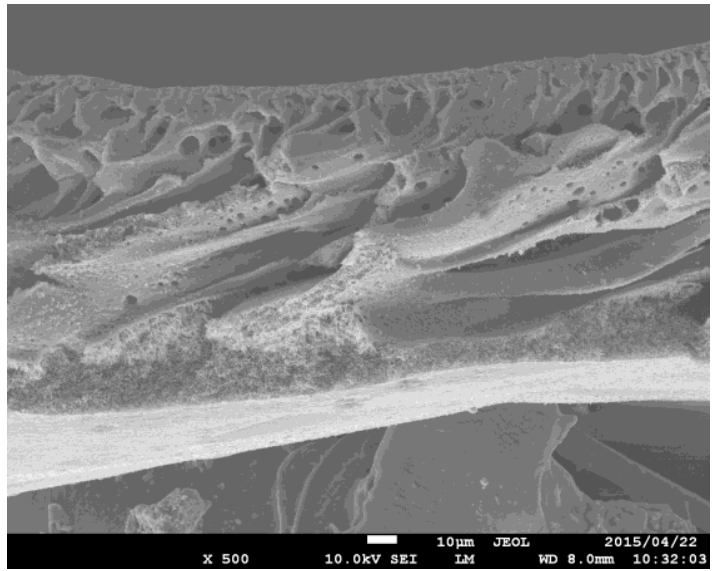

( a )

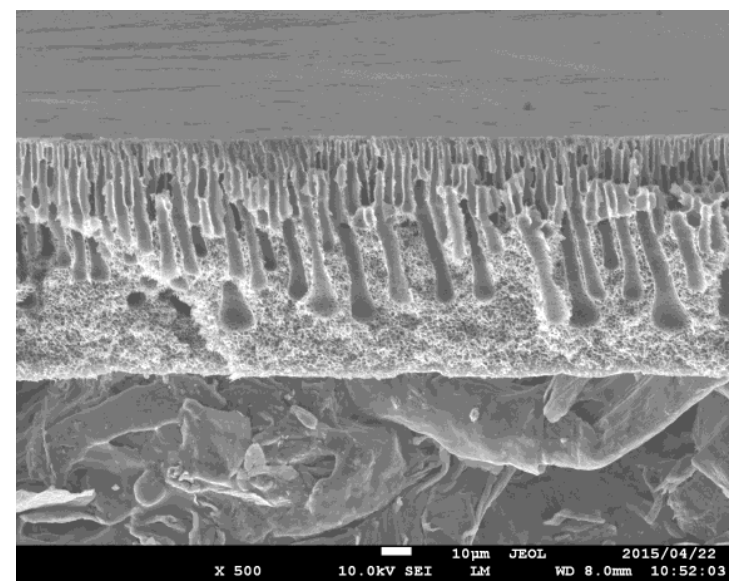

(b)

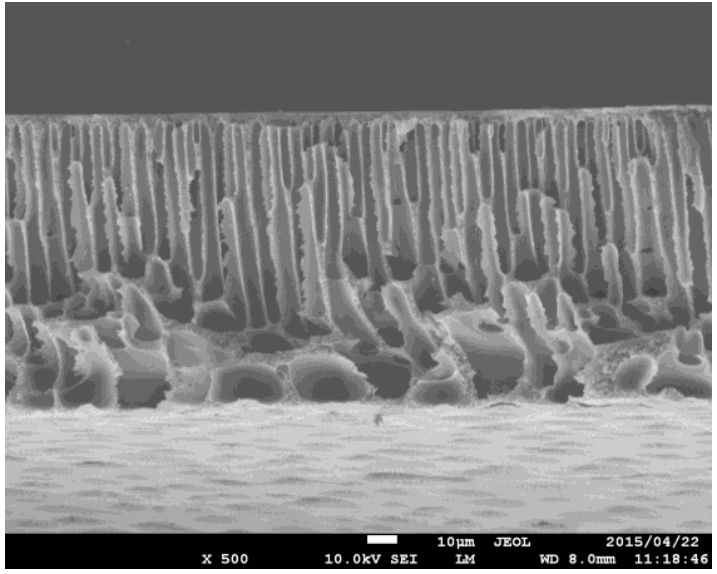

(c)

Fig. 2. SEM images of top surface membranes: PES-NMP (a); PES-DMF (b); PES-DMSO(c).

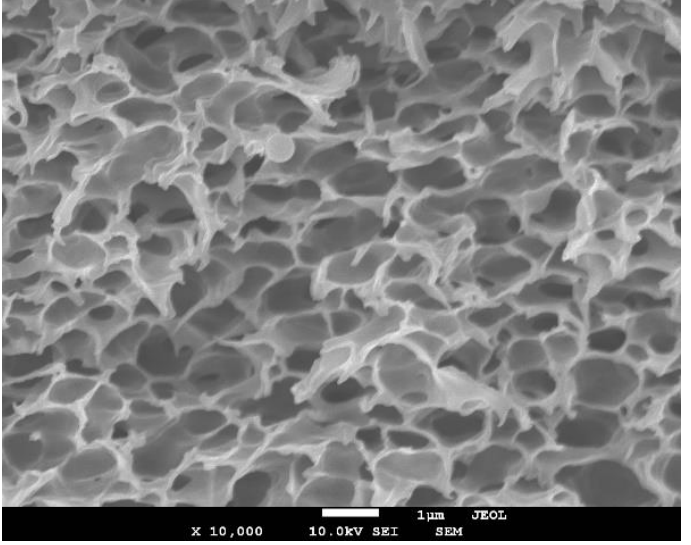

( a )

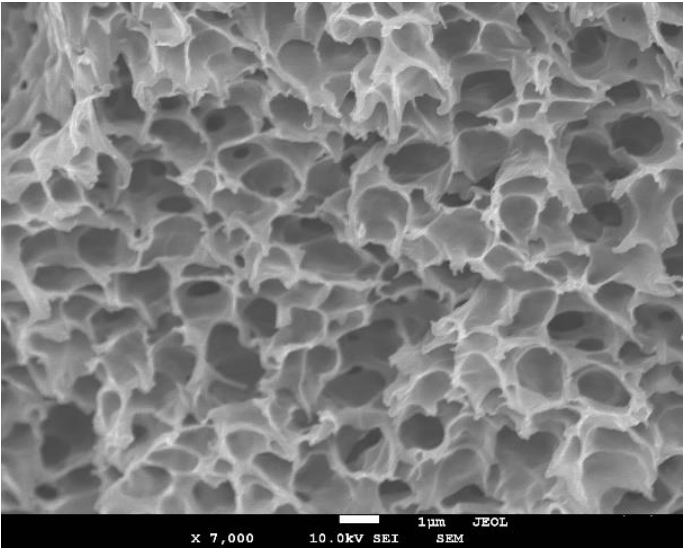

( b )

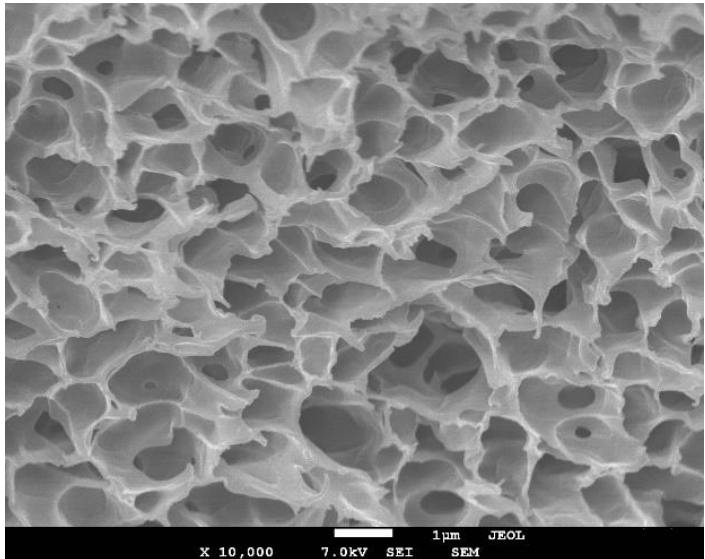

(c)

Fig. 3. SEM images of sponge structure: PES-NMP (a); PESDMF (b); PES-DMSO(c).

Figure 3 is the SEM image of the exclusive observation of membrane pores in the wall of macrovoid for all polymer system. Each fabricated membrane has pore structure like honey comb distributed in all membrane area. The figure shows that the pore sizes of PES/DMSO membrane was the largest than other systems. Comparing three polymer system with different solvent, the PES DMSO membrane showed more porous It is showed that the PES/DMSO membranes has the highest pore density than others. In sum, the pore structure of membrane was greatly affected by the kind of solvents. 


\subsection{Filtration performance}

It is already known that the membrane pore structures has great impact on the water permeation during filtration process $[9,10]$. So, a dead-end filtration module was used to observe the pure water permeability (PWP) of the formed membrane. The effect of membrane structure on the filtration performance process are given in Figure 4. Overall, PES/DMSO membrane showed the highest water permeability. The same result is reported by Arthanareeswaran and Starov [4] that PES-DMSO system has highest permeation flux that PES-NMP and PES-DMF systems. Hasbullah et al. [5] also has the same result that PES-DMF system has higher water permeability than PES-NMP system.

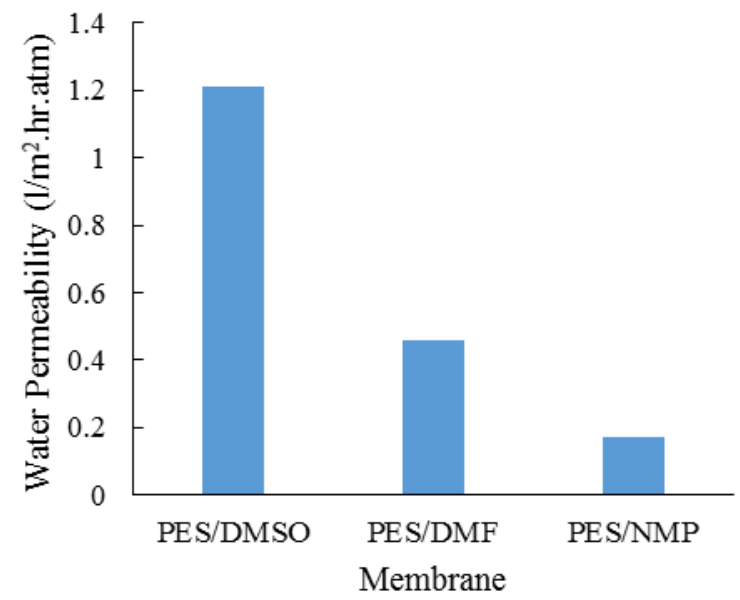

Fig. 4. Filtration performance of various membrane systems.

\section{Conclusions}

PES membrane with different polymer solution have been successfully prepared via induced phased separation method. The PES-DMSO membrane has more macrovoid structure. The changing of membrane structure determines the pure water permeability (PWP) of fabricated membrane. PES-DMSO membrane has the highest water permeability than other systems.

Acknowledgment for the Ministry of Research, Technology, and Higher Education, Indonesia for financial support of this research.

\section{References}

1. A. Rahimpour, S.S. Madaeni, "Improvement of performance and surface properties of nano-porous polyethersulfone (PES) membrane using hydrophilic monomers as additives in the casting solution," J. Memb. Sci. 360 371-379 (2010)

2. N. Arahman, S. Mulyati, M.R. Lubis, F. Razi, R. Takagi, H. Matsuyama, "Modification of polyethersulfone hollow fiber membrane with different polymeric additives," Membr. Water Treat. 4 355-365 (2016)
3. Q. Zhao, C. Liu, J. Liu, Y. Zhang, "Development of a novel polyethersulfone ultrafiltration membrane with antibacterial activity and high flux containing halloysite nanotubes loaded with lysozyme," RSC Adv. 5 38646-38653 (2015)

4. S. Velu, L. Muruganandam, G. Arthanareeswaran, "Effect of Solvents on Performance of Polyethersulfone Ultrafiltration Membranes for Separation of Metal Ions," Int. J. Chem. Anal. Sci. 2 82-86 (2011)

5. G. Arthanareeswaran, V.M. Starov, "Institutional Repository Effect of solvents on performance of polyethersulfone ultrafiltration membranes: investigation of metal ion separations," Desalination. 267 57-63 (2010)

6. H. Hasbullah, A. Ismail, B. Ng, M. Abdullah, "Effects of Solvent Types on Structure and Performance of Polyethersulfone Nanofiltration Membrane in Monovalent and Divalent Ions Separation," ICCBPE/SOMChe. 869-874 (2005)

7. H. Arahman, N., Maruyama, T, Sotani, T, Matsuyama, "Effect of Hypochlorite Treatment on Performance of Hollow Fiber Membrane Prepared from Polyethersulfone/N-Methyl-2-Pyrrolidone/ Tetronic 1307 Solution," 687-694 (2008)

8. N. Arahman, "Modification of the morphology of the poly (ether sulfone) membrane prepared by dry phase inversion technique," Int. J. Appl. Eng. Res. 9 10453-10462 (2014)

9. H. Susanto, M. Ulbricht, "Characteristics, performance and stability of polyethersulfone ultrafiltration membranes prepared by phase separation method using different macromolecular additives," J. Memb. Sci. 327 125-135 (2009)

10. H. Matsuyama, Y. Takida, T. Maki, M. Teramoto, "Preparation of porous membrane by combined use of thermally induced phase separation and immersion precipitation," 43 5243-5248 (2002) 